\title{
A Case of Giant Molluscum Contagiosum in an Immunocompetent Child
}

\section{Keywords: Giant molluscum contagiosum; Immunocompetent;} Child

A 4-year-old boy presented with an asymptomatic, erythematous mass on the abdomen. His mother said that the lesion developed a few months ago and have been gradually increasing in size. His weight was $19 \mathrm{~kg}$, height was $95 \mathrm{~cm}$ and showed normal development. The patient did not show any signs of immunodeficiencies. The past history and family history was unremarkable. On close examination, a solitary, $0.5 \mathrm{~cm}$-sized, pedunculated, erythematous mass lesion was present on the abdomen (Figure 1). Multiple, tiny, umbilicated, skincolored papules were also noticed on the trunk and extremities. Punch biopsy was performed at the erythematous lesion. Histopathologic examination showed lobular epithelial hyperplasia with central keratinization. Diffuse lymphocytic infiltration and intracytoplasmic inclusion bodies were observed, which was consistent with molluscum

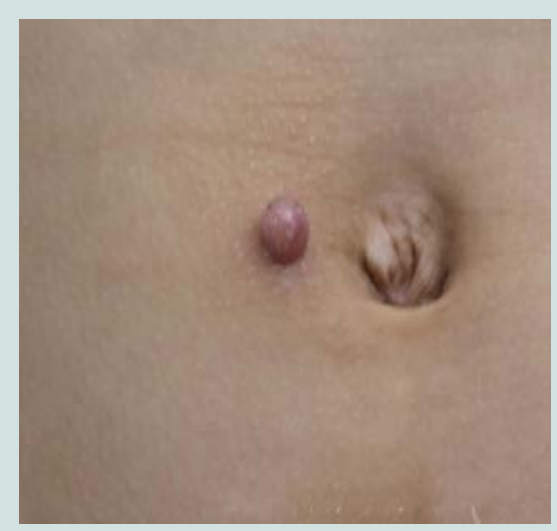

Figure 1: Solitary, $0.5 \mathrm{~cm}$-sized, erythematous, pedunculated nodule on the abdomen.



Figure 2: (A) Circumscribed multilobular epithelial proliferation with central keratinization. (B) Diffuse lymphocytic infiltration and intracytoplasmic inclusions were also observed.

\section{Journal of}

\section{Clinical \& Investigative} Dermatology

\section{Seok Hoon Moon, Sang Hyun Cho, Jeong Deuk Lee} and Hei Sung Kim*

Department of Dermatology, Incheon St. Mary's Hospital, College of Medicine, Catholic University of Korea, Korea

\section{*Address for Correspondence}

Hei Sung Kim, Department of Dermatology, Incheon St. Mary's Hospital, College of Medicine, Catholic University of Korea, 56 Dongsu-ro, Bupyeong-gu, Incheon 403-720, Korea, Tel: 82-32-280-5700; Fax: 82-2506-9514; E-mail: hazelkimhoho@gmail.com

Submission: 08 December, 2016

Accepted: 17 December, 2016

Published: 22 December, 2016

Copyright: @ 2016 Moon SH, et al. This is an open access article distributed under the Creative Commons Attribution License, which permits unrestricted use, distribution, and reproduction in any medium, provided the original work is properly cited.

contagiosum (Figure 2).

Molluscum contagiosum is a self-limiting skin infection caused by a member of the molluscum contagiosum virus (MCV1-4), the poxvirus family. Incidence peaks in pre-school children and reports of infection have also been made in immunocompromised adults. The virus directly enters into the epidermis from skin defect or can be disseminated by simple contact. The disease is self-limiting in immunocompetent individuals but severe and prolonged cases have been reported in Human Immunodeficiency Virus (HIV) infected patients [1]. Clinically, it is characterized by 1 to $3 \mathrm{~mm}$ sized, skin-colored, umbilicated papules that usually appear on the face, trunk, and limbs [2]. There is typical central umbilication from which cheesy kaeratinous material can be expressed. The incubation period is around 2 weeks. Widespread, multiple MC lesions are more common in children. Atypical presentations, such as facial, large, hyperkeratotic lesions can be seen and are more frequent in immunocompromised adults.

Molluscum contagiosum with a diameter greater than $0.5-1 \mathrm{~cm}$ is classified as a 'Giant MC'. The giant atypical MC rarely occurs in healthy individuals. The giant variant of $\mathrm{MC}$ can be a single lesion and as they mostly occur at the eyelids, scalp, and soles, they can be easily confused with other conditions like basal cell carcinoma, abscess, furuncle, and various adenomas. They usually occur in conditions with altered immunity as atopic dermatitis, patients on immune-suppressive therapy, leukemias, Wiskott-Aldrich syndrome and AIDS [3].

The diagnosis of MC is usually made based on the typical clinical findings. However, atypical forms may require biopsy to confirm the diagnosis. The treatment does not differ the classic forms of MC. Surgical methods including curettage, electrodessication, cryotherapy, and laser surgery are often adopted. Topical cytodestructive agents including cantharadin, iodine, lactic acid, tretinoin, trichloroacetic acids and systemic chemotherapeutic agents including cidofovir, interferon, imiquimod can be considered as alternative treatment options [2]. In our case, the giant MC lesion was completely removed 
Citation: Moon SH, Cho SH, Lee JD, Kim HS. A Case of Giant Molluscum Contagiosum in an Immunocompetent Child. J Clin Investigat Dermatol. 2016;4(2): 2

ISSN: 2373-1044

with a $6 \mathrm{~mm}$ punch biopsy and there has been no sign of recurrence at 6 months follow-up.

Solitary giant MC, although a rare condition in an immunocompetent patient, should be suspected if typical clinical presentations such as a central umbilication is found. Complete removal with a punch and concurrent biopsy is considered as one of the best diagnostic and therapeutic option for giant MC, especially when the diagnosis is uncertain.

\section{References}

1. Vora RV, Pilani AP, Kota RK (2015) Extensive giant molluscum contagiosum in a HIV positive Patient. J Clin Diagn Res 9: WD01-2.

2. Karadag AS, Karadag R, Bilgili SG, Calka O, Demircans YT (2013) Giant molluscum contagiosum in an immunocompetent child. J Pak Med Assoc 63 778-779.

3. Vardhan P, Goel S, Goyal G, Kumar N (2010) Giant molluscum contagiosum presenting as lid tumor in an immunocompetent child. Indian $\mathrm{J}$ Ophthalmol 58: $236-238$.

\section{Acknowledgements}

Funding Sources: This study was supported by a grant of the Korean Healthcare technology R\&D project, Ministry of Health \& Welfare, Republic of Korea (Grant No.: NH15C0105). 